\title{
P01-4-5 Poster session
}

\section{Possible role of SERPINA1 expression in adipocyte function under endoplasmic reticulum stress}

\section{Kazuhiro Tamura ${ }^{1}$, Akihito Kuroda ${ }^{1}$, Yukari Ando ${ }^{1}$, Mikihiro Yoshie ${ }^{1}$, Naoko Kuwabara ${ }^{1}$, Eiichi Tachikawa ${ }^{1}$, Takeshi Matsutani ${ }^{2}$}

${ }^{I}$ Endocrine \& Neural Pharmacology, Tokyo Univ. of Pharmacy \& Life Sciences, Japan, ${ }^{2}$ Nihon Medical School, Japan

Obesity-induced endoplasmic reticulum (ER) stress contributes to low-grade chronic inflammation, in adipose tissue and results in metabolic disorders such as diabetes mellitus and dyslipidemia. Sepsis displays a major cause of death on intensive care units and also contributes substantially to mortality after surgery. The incidence of severe sepsis in obesity patients is higher than that in non-obesity patients. Sepsis is a complex acute inflammatory disease, and the inflammatory response is characterized by the systemic circulation of inflammatory cytokines. However, changes in adipocyte function under ER stress and the role of adipose tissues in sepsis remains understood. Our previous study revealed that alpha1antitrypsin (SERPINA1: A1AT), which exhibits tissue protective action and the activity of serine protease inhibiter, is highly expressed in adipose tissues and adipocytes. We determined here the importance of adipocyte A1AT expression for adipocyte homeostasis under ER stress. To address this issue, the ER stress activator thapsigargin was treated to differentiated SW872 adipocytes after A1AT siRNA treatment. Thapsigargin markedly increased the basal levels of proinflammatory adipokines (IL-1beta, IL-6, and IL-8) expression. Knocking down A1AT enhanced the expression of thapsigargin-stimulated adipokines. Furthermore, the knockdown of A1AT reduced insulin-induced phosphorylation of Akt, whereas stimulated TNF-alpha-triggered pro-inflammatory cytokines. These findings indicate the possibility that endogenous A1AT may negatively regulate inflammatory mechanism to inhibit excessive pro-inflammatory adipokine expression and play a protective role in adipose tissue dysfunction mediated through ER stress activation. 